\title{
A Mathematical Model for Visco-Ferromagnetic Materials
}

\author{
Giovambattista Amendola $^{1}$, Mauro Fabrizio ${ }^{2, *}$ and John Murrough Golden ${ }^{3}$ \\ ${ }^{1}$ Department of Mathematics, University of Pisa, Italy \\ ${ }^{2}$ Department of Mathematics, University of Bologna, Italy \\ ${ }^{3}$ School of Mathematical Sciences, Technological University, Dublin, Ireland
}

Received: 12 Feb. 2020, Revised: 22 Mar. 2020, Accepted: 22 Apr. 2020

Published online: 1 Oct. 2020

\begin{abstract}
Visco-ferromagnetic materials represented by non-local constitutive equation are considered in the paper. We use fractional derivatives in order to describe memory and spatial effects. Also, thermodynamic principles are formulated and studied.
\end{abstract}

Keywords: Visco-ferromagnetism, non-local fractional derivative, dissipation of the electromagnetic energy.

\section{Introduction}

Ferromagnetic materials are perhaps best introduced by recalling a well-known property associated with them. After an intense magnetization by applying an external magnetic field $\mathbf{H}$, they remain magnetized for a long time even if the field $\mathbf{H}$ goes to zero; thus, the materials become permanent magnets.

Another feature, which will be modeled later, is that for an increasing magnetic field $\mathbf{H}$, the associated magnetic induction $\mathbf{B}$ exhibits a weaker rate of change.

The various sections below deal with the following topics. In Section 2, we recall Maxwell's (general) equations and some classical constitutive equations, while, in Section 3, visco-ferromagnetic materials are introduced. Then, in Section 4 , we present a more complex constitutive equation applying to non-local visco-ferromagnetic materials. The Maxwell differential system for such materials with appropriate initial and boundary conditions are presented in Section 5, while Section 6 contains thermodynamic restrictions related to stability conditions for these differential problems. Finally, in Section 7, a new type of non-local fractional derivative is introduced to model non-local visco-ferromagnetic materials. These involve non-singular kernels in both space and time.

\section{Balance equation in electromagnetism}

Let us introduce the following quantities: the electric field $\mathbf{E}(\mathbf{x}, t)$, the electric displacement $\mathbf{D}(\mathbf{x}, t)$, the magnetic field $\mathbf{H}(\mathbf{x}, t)$, the magnetic induction $\mathbf{B}(\mathbf{x}, t)$, the charge density $\rho(\mathbf{x}, t)$ and the electric current $\mathbf{J}(\mathbf{x}, t)$. These fields are defined in a smooth region $\Omega \subset \mathbb{R}^{3}$ and for a given time period $T$, so that $\mathbf{x} \in \Omega$ and $t \in T$. They must satisfy [1] Faraday's law and Maxwell's law:

$$
\begin{gathered}
\nabla \times \mathbf{E}=-\frac{\partial \mathbf{B}}{\partial t}, \\
\nabla \times \mathbf{H}=\mathbf{J}+\frac{\partial \mathbf{D}}{\partial t},
\end{gathered}
$$

together with Gauss's law and the conservation of magnetic flux, given by

$$
\nabla \cdot \mathbf{D}=\rho, \quad \nabla \cdot \mathbf{B}=0,
$$

\footnotetext{
*Corresponding author e-mail: mauro.fabrizio@unibo.it
} 
and the charge balance law in the following local form

$$
\frac{\partial \rho}{\partial t}+\nabla \cdot \mathbf{J}=0
$$

which is the continuity equation, in a particular choice of units.

The properties of materials are defined by suitable constitutive equations, which characterize their behaviour. A particular classical choice of constitutive equations is given by

$$
\mathbf{D}(\mathbf{x}, t)=\hat{\mathbf{D}}(\mathbf{E}(\mathbf{x}, t), \mathbf{H}(\mathbf{x}, t)), \quad \mathbf{B}(\mathbf{x}, t)=\hat{\mathbf{B}}(\mathbf{E}(\mathbf{x}, t), \mathbf{H}(\mathbf{x}, t)),
$$

where both fields $\mathbf{D}(\mathbf{x}, t)$ and $\mathbf{B}(\mathbf{x}, t)$ depend on the fields $\mathbf{E}(\mathbf{x}, t)$ and $\mathbf{H}(\mathbf{x}, t)$ at the same space and time points. Moreover, the current density $\mathbf{J}$ can be represented by

$$
\mathbf{J}(\mathbf{x}, t)=\hat{\mathbf{J}}(\mathbf{E}(\mathbf{x}, t), \mathbf{H}(\mathbf{x}, t))
$$

These relations are local in both space and time. Below, we will consider constitutive equations which are non-local in space and also with memory, which can be regarded as non-local in time.

\section{Visco-ferromagnetic materials}

A model that describes a visco-ferromagnetic material as a linear system with a fractional derivative [2,3] is now presented. We propose the following constitutive equation

$$
\mathbf{B}(\mathbf{x}, t)=\frac{\mathbf{C}(\mathbf{x})}{\Gamma(1-\alpha)} \int_{a}^{t} \frac{\mathbf{H}_{\tau}(\mathbf{x}, \tau)}{(t-\tau)^{\alpha}} d \tau, \quad \mathbf{H}_{\tau}(\mathbf{x}, \tau)=\frac{\partial}{\partial \tau} \mathbf{H}(\mathbf{x}, \tau)
$$

where $\Gamma(1-\alpha)$ is the gamma function evaluated at $(1-\alpha), a \leq 0$, and the quantity $\mathbf{C}(\mathbf{x})$ is a second order positive tensor, defined for any point $\mathbf{x} \in \Omega$; moreover, the field $\mathbf{H}(\mathbf{x}, \tau)$ is assumed to be zero for any $\tau<a$. Defining the $\alpha-$ Caputo fractional derivative by

$$
{ }_{C} D_{t}^{\alpha} \mathbf{H}(\mathbf{x}, t)=\frac{1}{\Gamma(1-\alpha)} \int_{a}^{t} \frac{\mathbf{H}_{\tau}(\mathbf{x}, \tau)}{(t-\tau)^{\alpha}} d \tau,
$$

where $\alpha \in[0,1]$ denotes its degree, then equation (5) can be written as

$$
\mathbf{B}(\mathbf{x}, t)=\mathbf{C}(\mathbf{x})_{C} D_{t}^{\alpha} \mathbf{H}(\mathbf{x}, t) .
$$

Taking $a$ in (5) to be $-\infty$, we obtain

$$
\begin{aligned}
\mathbf{B}(\mathbf{x}, t) & =\frac{1}{\Gamma(1-\alpha)} \int_{-\infty}^{t} \mathbf{C}(\mathbf{x}) \frac{\mathbf{H}_{\tau}(\mathbf{x}, \tau)}{(t-\tau)^{\alpha}} d \tau \\
& =-\frac{\alpha}{\Gamma(1-\alpha)} \int_{-\infty}^{t} \mathbf{C}(\mathbf{x}) \frac{\mathbf{H}^{(r)}(\mathbf{x}, \tau)}{(t-\tau)^{\alpha+1}} d \tau, \\
\mathbf{H}^{(r)}(\mathbf{x}, \tau) & =\mathbf{H}(\mathbf{x}, \tau)-\mathbf{H}(\mathbf{x}, t),
\end{aligned}
$$

which is more convenient for some problems. The quantity $\mathbf{H}^{(r)}$ is the relative history of the magnetic field. Note that equation (8) can be written in the standard form of a constitutive equation for a material with memory

$$
\begin{aligned}
\mathbf{B}(\mathbf{x}, t) & =\int_{-\infty}^{t} \mathbf{G}(\mathbf{x}, t-\tau) \mathbf{H}_{\tau}(\mathbf{x}, \tau) d \tau \\
& =\int_{-\infty}^{t} \mathbf{G}^{\prime}(\mathbf{x}, t-\tau) \mathbf{H}^{(r)}(\mathbf{x}, \tau) d \tau \\
\mathbf{G}(\mathbf{x}, s) & =\frac{1}{\Gamma(1-\alpha)} \frac{\mathbf{C}(\mathbf{x})}{s^{\alpha}} .
\end{aligned}
$$

This is a degenerate material in the sense that $\mathbf{G}(\mathbf{x}, 0)$ is infinite and $\mathbf{G}(\mathbf{x}, \infty)$ is zero. 
There are various free energies associated with such a constitutive equation, all of them quadratic functionals. Denoting a particular free energy by $\psi(\mathbf{x}, t) \geq 0$, we must have the fundamental dissipation requirement, given by

$$
\frac{\partial}{\partial t} \psi(\mathbf{x}, t) \leq \mathbf{B}(\mathbf{x}, t) \cdot \mathbf{H}_{t}(\mathbf{x}, t)
$$

Another way of stating this requirement is to introduce the rate of dissipation $\mathscr{D}(\mathbf{x}, t) \geq 0$, putting

$$
\frac{\partial}{\partial t} \psi(\mathbf{x}, t)+\mathscr{D}(\mathbf{x}, t)=\mathbf{B}(\mathbf{x}, t) \cdot \mathbf{H}_{t}(\mathbf{x}, t)
$$

This constraint will be discussed further below.

One particular choice is the Graffi-Volterra free energy, which, after a change of variable, is given by

$$
\begin{aligned}
\psi(t) & =-\frac{1}{2} \int_{0}^{\infty} \mathbf{G}^{\prime}(\mathbf{x}, s) \mathbf{H}^{(r)}(\mathbf{x}, t-s) \cdot \mathbf{H}^{(r)}(\mathbf{x}, t-s) d s \\
& =\frac{\alpha}{2 \Gamma(1-\alpha)} \int_{0}^{\infty} \mathbf{C}(\mathbf{x}) \mathbf{H}^{(r)}(\mathbf{x}, t-s) \cdot \mathbf{H}^{(r)}(\mathbf{x}, t-s) \frac{d s}{s^{\alpha+1}}
\end{aligned}
$$

with associated rate of dissipation

$$
\begin{aligned}
\mathscr{D}(\mathbf{x}, t) & =\frac{1}{2} \int_{0}^{\infty} \mathbf{G}^{\prime \prime}(\mathbf{x}, s) \mathbf{H}^{(r)}(\mathbf{x}, t-s) \cdot \mathbf{H}^{(r)}(\mathbf{x}, t-s) d s \\
& =\frac{\alpha(\alpha+1)}{2 \Gamma(1-\alpha)} \int_{0}^{\infty} \mathbf{C}(\mathbf{x}) \mathbf{H}^{(r)}(\mathbf{x}, t-s) \cdot \mathbf{H}^{(r)}(\mathbf{x}, t-s) \frac{d s}{s^{\alpha+2}} .
\end{aligned}
$$

The equilibrium free energy is zero in this context, which is related to the fact that there is no equilibrium term in the constitutive equation (8), and ultimately to the property $\mathbf{G}(\mathbf{x}, \infty)=\mathbf{0}$. Moreover, the following conditions

$$
\mathbf{G}^{\prime}(s) \leq 0, \quad \mathbf{G}^{\prime \prime}(s) \geq 0,
$$

for all non-negative values of $s$, must be obeyed if $\psi(t)$ is to be a free energy. These clearly hold in the present case.

This functional is the only single-integral quadratic functional related to the constitutive equation (8) and having the required properties of a free energy. Other free energies must be double-integral quadratic functionals.

\section{Non-local visco-ferromagnetic materials}

In order to study non-local visco-ferromagnetic materials, we introduce the fractional operator $\mathscr{M}_{\mathbf{x}}^{\beta}$ of order $\beta \in\left(\frac{1}{2}, 1\right)$ of a function $\mathbf{F}(\mathbf{x})[4,5]$ defined by

$$
\mathscr{M}_{\mathbf{x}}^{\beta} \mathbf{F}(\mathbf{x})=\frac{\beta \pi^{-\frac{\beta}{2}}}{1-\beta} \int_{\Omega} \mathbf{F}(\mathbf{y}) e^{-\frac{\beta^{2}}{1-\beta^{2}}(\mathbf{x}-\mathbf{y})^{2}} d \mathbf{y} .
$$

Then, we consider a new constitutive equation for ferromagnetic induction, expressed in terms of such a fractional operator, where $\mathbf{F}(\mathbf{x})$ is replaced by the magnetic field $\mathbf{H}(\mathbf{x}, \tau)$, giving the following equation

$$
\begin{aligned}
\mathbf{B}(\mathbf{x}, t) & =\mathbf{C}(\mathbf{x}) \mathscr{M}_{\mathbf{x}}^{\beta} D_{t}^{\alpha} \mathbf{H}(\mathbf{x}, t) \\
& =\frac{\beta \pi^{-\frac{\beta}{2}}}{(1-\beta) \Gamma(1-\alpha)} \int_{\Omega} \int_{a}^{t} \frac{1}{(t-\tau)^{\alpha}} \mathbf{C}(\mathbf{x}) \mathbf{H}_{\tau}(\mathbf{y}, \tau) e^{-\frac{\beta^{2}}{1-\beta^{2}}(\mathbf{x}-\mathbf{y})^{2}} d \tau d \mathbf{y}
\end{aligned}
$$

where the scalar $\alpha$, the degree of the Caputo fractional derivative here denoted by $D_{t}^{\alpha}$, for this problem will be assumed to be in the interval $\left(0, \frac{1}{2}\right)$.

Let $a=0$ and $\mathbf{H}(\mathbf{x}, \tau)=\mathbf{H}_{0}(\mathbf{x})+\mathbf{H}_{1}(\mathbf{x}) \tau$ in the time interval $[0, t]$, where both $\mathbf{H}_{0}(\mathbf{x}), \mathbf{H}_{1}(\mathbf{x})$ are bounded in $\Omega$; thus, the magnetic field is bounded and increasing linearly in this interval.

We have

$$
\mathbf{B}(\mathbf{x}, t)=\frac{\beta \pi^{-\frac{\beta}{2}}}{(1-\beta) \Gamma(1-\alpha)} \int_{\Omega} \int_{0}^{t} \frac{\mathbf{C}(\mathbf{x}) \mathbf{H}_{1}(\mathbf{y})}{(t-\tau)^{\alpha}} e^{-\frac{\beta^{2}}{1-\beta^{2}}(\mathbf{x}-\mathbf{y})^{2}} d \tau d \mathbf{y} .
$$


The time integral can be carried out, so that

$$
\mathbf{B}(\mathbf{x}, t)=\frac{\beta \pi^{-\frac{\beta}{2}} t^{1-\alpha}}{(1-\beta) \Gamma(2-\alpha)} \int_{\Omega} \mathbf{C}(\mathbf{x}) \mathbf{H}_{1}(\mathbf{y}) e^{-\frac{\beta^{2}}{1-\beta^{2}}(\mathbf{x}-\mathbf{y})^{2}} d \mathbf{y} .
$$

Thus, if the magnetic field $\mathbf{H}(\mathbf{x}, \tau)$ increases linearly in $\tau$, the ferromagnetic induction $\mathbf{B}(\mathbf{x}, t)$ exhibits a weaker rate of increase, as noted in the Introduction.

\section{The differential problem for non-local ferromagnetic materials}

Consider the classical electromagnetic differential system

$$
\begin{array}{lll}
\frac{\partial \mathbf{D}(\mathbf{x}, t)}{\partial t}=\nabla \times \mathbf{H}(\mathbf{x}, t)-\mathbf{J}(\mathbf{x}, t), & \nabla \cdot \mathbf{D}(\mathbf{x}, t)=\rho(\mathbf{x}), \\
\frac{\partial \mathbf{B}(\mathbf{x}, t)}{\partial t}=-\nabla \times \mathbf{E}(\mathbf{x}, t), & \nabla \cdot \mathbf{B}(\mathbf{x}, t)=0,
\end{array}
$$

where the electric current $\mathbf{J}(\mathbf{x}, t)$ is a given function, while the electric displacement $\mathbf{D}(\mathbf{x}, t)$ and the electric field $\mathbf{E}(\mathbf{x}, t)$ are related by a constitutive equation of the form

$$
\mathbf{D}(\mathbf{x}, t)=\boldsymbol{\varepsilon}(\mathbf{x}) \mathbf{E}(\mathbf{x}, t),
$$

where $\boldsymbol{\varepsilon}(\mathbf{x})$ is a positive second order tensor, assumed to be given by $\varepsilon_{0}(\mathbf{x}) \mathbf{1}$, where $\mathbf{1}$ is the identity tensor and $\varepsilon_{0}$ is a scalar quantity.

Thus, by using equations (19), (20) and (21) with $\varepsilon_{0}(\mathbf{x})=1$, we obtain

$$
\frac{\partial^{2} \mathbf{B}(\mathbf{x}, t)}{\partial t^{2}}=-\nabla \times \nabla \times \mathbf{H}(\mathbf{x}, t)+\nabla \times \mathbf{J}(\mathbf{x}, t) .
$$

We associate with this equation the initial conditions

$$
\mathbf{H}(\mathbf{x}, 0)=\mathbf{H}_{0}(\mathbf{x}),\left.\quad \dot{\mathbf{H}}(\mathbf{x}, t)\right|_{t=0}=\dot{\mathbf{H}}_{0}(\mathbf{x})
$$

and the boundary condition

$$
\mathbf{H}(\mathbf{x}, t) \times\left.\mathbf{n}(\mathbf{x})\right|_{\partial \Omega}=\mathbf{0}
$$

where $\mathbf{n}(\mathbf{x})$ denotes the unit normal to the boundary $\partial \Omega$ of $\Omega$.

Now, we observe that the tensor $\mathbf{B}(\mathbf{x}, t)$, defined by (16), in which the field $\mathbf{H}(\mathbf{x}, t)$ is the solution of the problem (22), (23) and (24), satisfies the condition

$$
\lim _{t \rightarrow \infty} \mathbf{B}(\mathbf{x}, t)=\mathbf{B}_{\infty}(\mathbf{x}) \neq \mathbf{0},
$$

where $\mathbf{B}_{\infty}(\mathbf{x})$ denotes the ferromagnetic field depending of the initial and the boundary conditions. The electric current $\mathbf{J}(\mathbf{x})$ is assumed to vanish.

\section{Energy and dissipation law}

In this section, because it is well known that there is a strong connection between thermodynamic restrictions and stability conditions [6], we state the Dissipation Law for simple materials related to the differential system (19), (20), and determine the form of a particular free energy $\psi(\mathbf{x}, t)$ for this system.

Dissipation Law. For any electromagnetic simple material, there exists a state function $\psi(\mathbf{x}, t)$, called a free energy, such that

$$
\frac{\partial}{\partial t} \psi(\mathbf{x}, t) \leq \mathbf{B}(\mathbf{x}, t) \cdot \mathbf{H}_{t}(\mathbf{x}, t)
$$

with $\mathbf{B}(\mathbf{x}, t)=\mathbf{B}\left(\mathbf{x}, \mathbf{H}(\mathbf{x}, t), \mathbf{H}^{t}(\mathbf{x}, s)\right)$, where $\mathbf{H}^{t}(\mathbf{x}, s)$ denotes the past history of $\mathbf{H}(\mathbf{x}, t)$. 
It is interesting to observe, that in the case of non-simple materials, for which the field $\mathbf{B}(\mathbf{x}, t)$ can depend of all magnetic fields $\mathbf{H}(\mathbf{y}, t)$ on the domain $\Omega$, the total internal power, by using the expression (16) for the vector $\mathbf{B}$, assumes the following form

$$
\begin{aligned}
\int_{\Omega} \mathbf{B}(\mathbf{x}, t) \cdot \mathbf{H}_{t}(\mathbf{x}, t) d \mathbf{x} & =\int_{\Omega} \mathscr{M}_{\mathbf{x}}^{\beta} D_{t}^{\alpha}[\mathbf{C}(\mathbf{x}, \mathbf{y}) \mathbf{H}(\mathbf{x}, t)] \cdot \mathbf{H}_{t}(\mathbf{x}, t) d \mathbf{x} \\
& =\frac{\beta \pi^{-\frac{\beta}{2}}}{(1-\beta) \Gamma(1-\alpha)} \int_{\Omega}\left[\int_{\Omega} \int_{a}^{t} \mathbf{C}(\mathbf{x}, \mathbf{y}) \frac{\mathbf{H}_{\tau}(\mathbf{y}, \tau)}{(t-\tau)^{\alpha}} e^{-\frac{\beta^{2}}{1-\beta^{2}}(\mathbf{x}-\mathbf{y})^{2}} d \tau d \mathbf{y}\right] \cdot \mathbf{H}_{t}(\mathbf{x}, t) d \mathbf{x}
\end{aligned}
$$

where $\mathbf{C}(\mathbf{x}, \mathbf{y})$ is symmetric in $\mathbf{x}$ and $\mathbf{y}$ and positively defined in the sense that for any function $\mathbf{f}$ defined on $\Omega$, the integral

$$
\int_{\Omega} \int_{\Omega} \mathbf{f}(\mathbf{x}) \mathbf{C}(\mathbf{x}, \mathbf{y}) \cdot \mathbf{f}(\mathbf{y}) d \mathbf{x} d \mathbf{y}
$$

is non-negative, if it exists.

Taking into account (25), (26) and assuming $\mathbf{H}(\cdot, \tau)=\mathbf{0}$ for $\tau \leq a$, we can write the Second Law, expressed by (10), in the new form

$$
\begin{aligned}
\frac{\partial}{\partial t} \int_{\Omega} \psi(\mathbf{x}, t) d \mathbf{x} & \leq \int_{\Omega} \mathbf{B}(\mathbf{x}, t) \cdot \mathbf{H}_{t}(\mathbf{x}, t) d \mathbf{x} \\
& =\frac{\beta \pi^{-\frac{\beta}{2}}}{(1-\beta) \Gamma(1-\alpha)} \int_{\Omega} \int_{\Omega} \int_{-\infty}^{t} \mathbf{C}(\mathbf{x}, \mathbf{y}) \frac{[\mathbf{H}(\mathbf{y}, \tau)-\mathbf{H}(\mathbf{y}, t)]_{\tau}}{(t-\tau)^{\alpha}} e^{-\frac{\beta^{2}}{1-\beta^{2}}(\mathbf{x}-\mathbf{y})^{2}} d \tau d \mathbf{y} \\
\cdot \frac{\partial}{\partial t}[\mathbf{H}(\mathbf{x}, t)-\mathbf{H}(\mathbf{x}, \tau)] d \mathbf{x} &
\end{aligned}
$$

Hence, carrying out a time integration by parts, we obtain

$$
\begin{aligned}
& \frac{\partial}{\partial t} \int_{\Omega} \psi(\mathbf{x}, t) d \mathbf{x} \leq \int_{\Omega} \mathbf{B}(\mathbf{x}, t) \cdot \mathbf{H}_{t}(\mathbf{x}, t) d \mathbf{x} \\
&=\frac{\alpha \beta \pi^{-\frac{\beta}{2}}}{(1-\beta) \Gamma(1-\alpha)} \int_{\Omega} \int_{\Omega} \int_{-\infty}^{t} \mathbf{C}(\mathbf{x}, \mathbf{y}) \frac{\mathbf{H}(\mathbf{y}, t)-\mathbf{H}(\mathbf{y}, \tau)}{(t-\tau)^{\alpha+1}} \\
& \cdot \frac{\partial}{\partial t}[\mathbf{H}(\mathbf{x}, t)-\mathbf{H}(\mathbf{x}, \tau)] e^{-\frac{\beta^{2}}{1-\beta^{2}}(\mathbf{x}-\mathbf{y})^{2}} d \tau d \mathbf{y} d \mathbf{x} .
\end{aligned}
$$

In order to evaluate this quantity, let us consider the following derivative

$$
\begin{aligned}
& \frac{\partial}{\partial t} \frac{\alpha \beta \pi^{-\frac{\beta}{2}}}{2(1-\beta) \Gamma(1-\alpha)} \int_{\Omega} \int_{\Omega} \int_{-\infty}^{t} \frac{1}{(t-\tau)^{\alpha+1}}[\mathbf{H}(\mathbf{y}, t)-\mathbf{H}(\mathbf{y}, \tau)] \\
& \cdot \mathbf{C}(\mathbf{x}, \mathbf{y})[\mathbf{H}(\mathbf{x}, t)-\mathbf{H}(\mathbf{x}, \tau)] e^{-\frac{\beta^{2}}{1-\beta^{2}}(\mathbf{x}-\mathbf{y})^{2}} d \tau d \mathbf{y} d \mathbf{x} \\
& =-\frac{\alpha \beta \pi^{-\frac{\beta}{2}}(\alpha+1)}{2(1-\beta) \Gamma(1-\alpha)} \int_{\Omega} \int_{\Omega} \int_{-\infty}^{t} \frac{1}{(t-\tau)^{\alpha+2}}[\mathbf{H}(\mathbf{y}, t)-\mathbf{H}(\mathbf{y}, \tau)] \\
& +\frac{\alpha \beta \pi^{-\frac{\beta}{2}}}{2(1-\beta) \Gamma(1-\alpha)} \int_{\Omega} \int_{\Omega} \int_{-\infty}^{t} \frac{1}{(t-\tau)^{\alpha+1}} \frac{\partial}{\partial t}\left\{[\mathbf{H}(\mathbf{y}, t)-\mathbf{H}, \mathbf{y})[\mathbf{H}(\mathbf{x}, t)-\mathbf{H}(\mathbf{x}, \tau)] e^{-\frac{\beta^{2}}{1-\beta^{2}}(\mathbf{x}-\mathbf{y})^{2}} d \tau d \mathbf{y} d \mathbf{x}\right. \\
& \cdot \mathbf{C}(\mathbf{x}, \mathbf{y})[\mathbf{H}(\mathbf{x}, t)-\mathbf{H}(\mathbf{x}, \tau)]\} e^{-\frac{\beta^{2}}{1-\beta^{2}}(\mathbf{x}-\mathbf{y})^{2}} d \tau d \mathbf{y} d \mathbf{x}
\end{aligned}
$$

where, under the assumption of sufficient regularity of the field $\mathbf{H}(\cdot, t)$ for any $t \in(a, b)$ where $a<0$ and $b>t$, we have applied $L^{\prime}$ Hopital's rule twice to show that the integrand function evaluated for $\tau=t$ vanishes.

We observe that the derivative of the scalar product in the last integral of (28) gives two integrals, which are equal and such that their sum coincides with the integral in (27), by virtue of the interchangeability of $\mathbf{x}$ and $\mathbf{y}$ in $\Omega$ and of the 
properties of $\mathbf{C}(\mathbf{x}, \mathbf{y})$. Thus, we can derive this sum from (28) and substitute it into (27), to obtain the following inequality

$$
\begin{aligned}
& \frac{\partial}{\partial t} \int_{\Omega} \psi(\mathbf{x}, t) d \mathbf{x} \equiv \frac{\partial}{\partial t} \psi(\Omega, t) \leq \int_{\Omega} \mathbf{B}(\mathbf{x}, t) \cdot \mathbf{H}_{t}(\mathbf{x}, t) d \mathbf{x} \\
& =\frac{\partial}{\partial t} \frac{\alpha \beta \pi^{-\frac{\beta}{2}}}{2(1-\beta) \Gamma(1-\alpha)} \int_{\Omega} \int_{\Omega} \int_{-\infty}^{t} \frac{1}{(t-\tau)^{\alpha+1}}[\mathbf{H}(\mathbf{y}, t)-\mathbf{H}(\mathbf{y}, \tau)] \\
& \cdot \mathbf{C}(\mathbf{x}, \mathbf{y})[\mathbf{H}(\mathbf{x}, t)-\mathbf{H}(\mathbf{x}, \tau)] e^{-\frac{\beta^{2}}{1-\beta^{2}}(\mathbf{x}-\mathbf{y})^{2}} d \tau d \mathbf{y} d \mathbf{x} \\
& +\frac{\alpha(\alpha+1) \beta \pi^{-\frac{\beta}{2}}}{2(1-\beta) \Gamma(1-\alpha)} \int_{\Omega} \int_{\Omega} \int_{-\infty}^{t} \frac{1}{(t-\tau)^{\alpha+2}}[\mathbf{H}(\mathbf{y}, t)-\mathbf{H}(\mathbf{y}, \tau)] \\
& \cdot \mathbf{C}(\mathbf{x}, \mathbf{y})[\mathbf{H}(\mathbf{x}, t)-\mathbf{H}(\mathbf{x}, \tau)] e^{-\frac{\beta^{2}}{1-\beta^{2}}(\mathbf{x}-\mathbf{y})^{2}} d \tau d \mathbf{y} d \mathbf{x}
\end{aligned}
$$

where $\psi(\Omega, t)$ denotes the global free energy.

Then, by comparing (29) with the relation

$$
\dot{\psi}(\Omega, t)+\mathscr{D}(\Omega, t)=\int_{\Omega} \mathbf{B}(\mathbf{x}, t) \cdot \dot{\mathbf{H}}(\mathbf{x}, t) d \mathbf{x}
$$

derived from (11) by integrating each of its three terms over $\Omega$, we obtain for $\psi(\Omega, t)$ the expression

$$
\psi(\Omega, t)=\frac{\alpha \beta \pi^{-\frac{\beta}{2}}}{2(1-\beta) \Gamma(1-\alpha)} \int_{\Omega} \int_{\Omega} \int_{-\infty}^{t} \frac{1}{(t-\tau)^{\alpha+1}}[\mathbf{H}(\mathbf{y}, t)-\mathbf{H}(\mathbf{y}, \tau)] \cdot \mathbf{C}(\mathbf{x}, \mathbf{y})[\mathbf{H}(\mathbf{x}, t)-\mathbf{H}(\mathbf{x}, \tau)] e^{-\frac{\beta^{2}}{1-\beta^{2}}(\mathbf{x}-\mathbf{y})^{2}} d \tau d \mathbf{y} d \mathbf{x}
$$

while the global dissipation $\mathscr{D}(\Omega, t)$ is given by

$$
\mathscr{D}(\Omega, t)=\frac{\alpha(\alpha+1) \beta \pi^{-\frac{\beta}{2}}}{2(1-\beta) \Gamma(1-\alpha)} \int_{\Omega} \int_{\Omega} \int_{-\infty}^{t} \frac{1}{(t-\tau)^{\alpha+2}}[\mathbf{H}(\mathbf{y}, t)-\mathbf{H}(\mathbf{y}, \tau)] \cdot \mathbf{C}(\mathbf{x}, \mathbf{y})[\mathbf{H}(\mathbf{x}, t)-\mathbf{H}(\mathbf{x}, \tau)] e^{-\frac{\beta^{2}}{1-\beta^{2}}(\mathbf{x}-\mathbf{y})^{2}} d \tau d \mathbf{y} d \mathbf{x}
$$

Remark. We observe that the limit $\lim _{\beta \rightarrow 1} \frac{\beta}{1-\beta} e^{-\frac{\beta^{2}}{1-\beta^{2}}(\mathbf{x}-\mathbf{y})^{2}}$ is proportional to the delta function $\delta\left((\mathbf{x}-\mathbf{y})^{2}\right)$. Thus, the free energy $\psi(\mathbf{x}, t)$ and the dissipation $\mathscr{D}(\mathbf{x}, t)$ are nonnegative for $\beta=1$, but also in a domain

$$
B_{\mathbf{x}}=\left\{\mathbf{y},(\mathbf{x}-\mathbf{y})^{2}<f(1-\beta), 0<<\beta<1\right\},
$$

where $f($.$) is a suitable positive function dependent on the material.$

\section{Constitutive equations for a new nonlocal visco-ferromagnetic material}

By using the time fractional derivative considered in [4] and denoted by $D_{t}^{(\alpha)}$, we introduce a new definition of non-local fractional derivative expressed by the following operator

$$
\mathscr{M}_{\mathbf{x}}^{\beta} D_{t}^{(\alpha)} \mathbf{u}(\mathbf{x}, t)=\frac{\beta \pi^{-\frac{\beta}{2}}}{(1-\beta)(1-\alpha)} \int_{\Omega} \int_{a}^{t} \frac{\partial \mathbf{u}(\mathbf{y}, \tau)}{\partial \tau} e^{-\frac{\beta^{2}}{1-\beta^{2}}(\mathbf{x}-\mathbf{y})^{2}-\frac{\alpha}{1-\alpha}(t-\tau)} d \tau d \mathbf{y}
$$

where $\alpha \in[0,1]$ and the function $\mathbf{u}(\cdot, \tau)$ is in a subset of $C(a, \infty)$, such that $a \in(-\infty, 0]$ and $\mathbf{u}(\cdot, a)=\mathbf{0}$. Also from this definition, we can prove that, when $\alpha=1$, we obtain the classical first time derivative, whereas, if $\alpha=0$, we have in (31) the function $\mathbf{u}(\cdot, t)$.

We can carry out an integration by parts in (31) to obtain

$$
\mathscr{M}_{\mathbf{x}}^{\beta} D_{t}^{(\alpha)} \mathbf{u}(\mathbf{x}, t)=\frac{\alpha \beta \pi^{-\frac{\beta}{2}}}{(1-\beta)(1-\alpha)^{2}} \int_{\Omega} \int_{-\infty}^{t}\left[\mathbf{u}(\mathbf{y}, t)-\mathbf{u}_{a}(\mathbf{y}, \tau)\right] e^{-\frac{\beta^{2}}{1-\beta^{2}}(\mathbf{x}-\mathbf{y})^{2}-\frac{\alpha}{1-\alpha}(t-\tau)} d \tau d \mathbf{y}
$$

where the function $\mathbf{u}_{a}$ is defined by

$$
\begin{array}{ll}
\mathbf{u}_{a}(\mathbf{y}, t)=\mathbf{u}(\mathbf{y}, t), & t \geq a, \\
\mathbf{u}_{a}(\mathbf{y}, t)=\mathbf{0}, & -\infty<t<a .
\end{array}
$$


Let us consider the case where $\mathbf{u}(\mathbf{x}, \tau)$ is the magnetic field $\mathbf{H}(\mathbf{x}, \tau)$ multiplied by $\mathbf{C}(\mathbf{x}, \mathbf{y})$.

A ferromagnetic body $\mathscr{B}$, on using the fractional derivative $(32)$, where $\mathbf{u}(\cdot, \tau)$ is substituted by $\mathbf{C}(\mathbf{x}, \mathbf{y}) \mathbf{H}(\cdot, \tau)$, is defined by the following constitutive equation

$$
\mathbf{B}(\mathbf{x}, t)=\frac{\alpha \beta \pi^{-\frac{\beta}{2}}}{(1-\beta)(1-\alpha)^{2}} \int_{\Omega} \int_{-\infty}^{t} \mathbf{C}(\mathbf{x}, \mathbf{y})\left[\mathbf{H}(\mathbf{y}, t)-\mathbf{H}_{a}(\mathbf{y}, \tau)\right] e^{-\frac{\beta^{2}}{1-\beta^{2}}(\mathbf{x}-\mathbf{y})^{2}-\frac{\alpha}{1-\alpha}(t-\tau)} d \tau d \mathbf{y},
$$

so that the state is defined by the pair $\left(\mathbf{H}(\cdot, t), \mathbf{H}^{t}(\cdot, s)\right)$, where $\mathbf{H}_{a}^{t}(\cdot, s)=\mathbf{H}_{a}(\cdot, t-s)$ with $s \in[0, \infty)$.

The time-dependent part of the kernel in (34) corresponds to the function $\mathbf{G}$ in (9) being proportional to a single decaying exponential, which in a viscoelastic context would be the Maxwell model [7].

Now, we can prove that equation (34) is compatible with the Dissipation Principle. Using the free energy $\psi\left(\mathbf{H}(\mathbf{x}, t), \mathbf{H}^{t}(\mathbf{x}, s)\right)$ we obtain the inequality

$$
\begin{aligned}
\int_{\Omega} \dot{\psi}(\mathbf{x}, t) d \mathbf{x} \equiv \dot{\psi}(\Omega, t) & \leq \int_{\Omega} \mathbf{B}(\mathbf{x}, t) \cdot \dot{\mathbf{H}}(\mathbf{x}, t) d \mathbf{x} \\
& =\frac{\alpha \beta \pi^{-\frac{\beta}{2}}}{(1-\beta)(1-\alpha)^{2}} \int_{\Omega}\left\{\int_{\Omega} \int_{-\infty}^{t} \mathbf{C}(\mathbf{x}, \mathbf{y})\left[\mathbf{H}(\mathbf{y}, t)-\mathbf{H}_{a}(\mathbf{y}, \tau)\right]\right. \\
& \left.\cdot \frac{\partial}{\partial t}\left[\mathbf{H}(\mathbf{x}, t)-\mathbf{H}_{a}(\mathbf{x}, \tau)\right] e^{-\frac{\beta^{2}}{1-\beta^{2}}(\mathbf{x}-\mathbf{y})^{2}-\frac{\alpha}{1-\alpha}(t-\tau)} d \tau d \mathbf{y}\right\} d \mathbf{x}
\end{aligned}
$$

This last quantity can be obtained by means of the following derivative

$$
\begin{aligned}
& \frac{\partial}{\partial t} \frac{\alpha \beta \pi^{-\frac{\beta}{2}}}{2(1-\beta)(1-\alpha)^{2}} \int_{\Omega} \int_{\Omega} \int_{-\infty}^{t} \mathbf{C}(\mathbf{x}, \mathbf{y})\left[\mathbf{H}(\mathbf{y}, t)-\mathbf{H}_{a}(\mathbf{y}, \tau)\right] \\
& \cdot\left[\mathbf{H}(\mathbf{x}, t)-\mathbf{H}_{a}(\mathbf{x}, \tau)\right] e^{-\frac{\beta^{2}}{1-\beta^{2}}(\mathbf{x}-\mathbf{y})^{2}-\frac{\alpha}{1-\alpha}(t-\tau)} d \tau d \mathbf{y} d \mathbf{x} \\
& =-\frac{\alpha^{2} \beta \pi^{-\frac{\beta}{2}}}{2(1-\beta)(1-\alpha)^{3}} \int_{\Omega} \int_{\Omega} \int_{-\infty}^{t}\left[\mathbf{H}(\mathbf{y}, t)-\mathbf{H}_{a}(\mathbf{y}, \tau)\right] \\
& +\frac{\alpha \beta \pi^{-\frac{\beta}{2}}}{2(1-\beta)(1-\alpha)^{2}} \int_{\Omega} \int_{\Omega} \int_{-\infty}^{t} \frac{\partial}{\partial t}\left\{\left[\mathbf{H}(\mathbf{y}, t)-\mathbf{H}(\mathbf{x}, \mathbf{y})\left[\mathbf{H}(\mathbf{x}, t)-\mathbf{H}_{a}(\mathbf{x}, \tau)\right] e^{-\frac{\beta^{2}}{1-\beta^{2}}(\mathbf{x}-\mathbf{y})^{2}-\frac{\alpha}{1-\alpha}(t-\tau)} d \tau d \mathbf{y} d \mathbf{x}\right.\right. \\
& \left.\cdot \mathbf{C}(\mathbf{x}, \mathbf{y})\left[\mathbf{H}(\mathbf{x}, t)-\mathbf{H}_{a}(\mathbf{x}, \tau)\right]\right\} e^{-\frac{\beta^{2}}{1-\beta^{2}}(\mathbf{x}-\mathbf{y})^{2}-\frac{\alpha}{1-\alpha}(t-\tau)} d \tau d \mathbf{y} d \mathbf{x} .
\end{aligned}
$$

By evaluating the derivative of the scalar product in the last integral of (36), we obtain two integrals, the sum of which gives the integral in (35), because of the properties of $\mathbf{C}(\mathbf{x}, \mathbf{y})$ and since $\mathbf{x}$ and $\mathbf{y}$ are interchangeable in $\Omega$.

Therefore, by substituting this sum, derived from (36), into (35), we have

$$
\begin{aligned}
& \frac{\partial}{\partial t} \int_{\Omega} \psi(\mathbf{x}, t) d \mathbf{x} \equiv \dot{\psi}(\Omega, t) \leq \int_{\Omega} \mathbf{B}(\mathbf{x}, t) \cdot \dot{\mathbf{H}}(\mathbf{x}, t) d \mathbf{x} \\
&= \frac{\partial}{\partial t} \frac{\alpha \beta \pi^{-\frac{\beta}{2}}}{2(1-\beta)(1-\alpha)^{2}} \int_{\Omega} \int_{\Omega} \int_{-\infty}^{t}\left[\mathbf{H}(\mathbf{y}, t)-\mathbf{H}_{a}(\mathbf{y}, \tau)\right] \\
& \cdot \mathbf{C}(\mathbf{x}, \mathbf{y})\left[\mathbf{H}(\mathbf{x}, t)-\mathbf{H}_{a}(\mathbf{x}, \tau)\right] e^{-\frac{\beta^{2}}{1-\beta^{2}}(\mathbf{x}-\mathbf{y})^{2}-\frac{\alpha}{1-\alpha}(t-\tau)} d \tau d \mathbf{y} d \mathbf{x} \\
&+\frac{\alpha^{2} \beta \pi^{-\frac{\beta}{2}}}{2(1-\beta)(1-\alpha)^{3}} \int_{\Omega} \int_{\Omega} \int_{-\infty}^{t}\left[\mathbf{H}(\mathbf{y}, t)-\mathbf{H}_{a}(\mathbf{y}, \tau)\right] \\
& \cdot \mathbf{C}(\mathbf{x}, \mathbf{y})\left[\mathbf{H}(\mathbf{x}, t)-\mathbf{H}_{a}(\mathbf{x}, \tau)\right] e^{-\frac{\beta^{2}}{1-\beta^{2}}(\mathbf{x}-\mathbf{y})^{2}-\frac{\alpha}{1-\alpha}(t-\tau)} d \tau d \mathbf{y} d \mathbf{x} .
\end{aligned}
$$
by

Thus, we observe that the equality obtained in (37) satisfies (30), with the global dissipation $\mathscr{D}(\Omega, t) \geq 0$ expressed

$$
\mathscr{D}(\Omega, t)=\frac{\alpha^{2} \beta \pi^{-\frac{\beta}{2}}}{2(1-\beta)(1-\alpha)^{3}} \int_{\Omega} \int_{\Omega} \int_{-\infty}^{t} \mathbf{C}(\mathbf{x}, \mathbf{y})\left[\mathbf{H}(\mathbf{y}, t)-\mathbf{H}_{a}(\mathbf{y}, \tau)\right] \cdot\left[\mathbf{H}(\mathbf{x}, t)-\mathbf{H}_{a}(\mathbf{x}, \tau)\right] e^{-\frac{\beta^{2}}{1-\beta^{2}}(\mathbf{x}-\mathbf{y})-\frac{\alpha}{1-\alpha}(t-\tau)} d \tau d \mathbf{y} d \mathbf{x}
$$


and the global free energy $\psi(\Omega, t)$ given by

$$
\psi(\Omega, t)=\frac{\alpha \beta \pi^{-\frac{\beta}{2}}}{2(1-\beta)(1-\alpha)^{2}} \int_{\Omega} \int_{\Omega} \int_{-\infty}^{t} \mathbf{C}(\mathbf{x}, \mathbf{y})\left[\mathbf{H}(\mathbf{y}, t)-\mathbf{H}_{a}(\mathbf{y}, \tau)\right] \cdot\left[\mathbf{H}(\mathbf{x}, t)-\mathbf{H}_{a}(\mathbf{x}, \tau)\right] e^{-\frac{\beta^{2}}{1-\beta^{2}}(\mathbf{x}-\mathbf{y})-\frac{\alpha}{1-\alpha}(t-\tau)} d \tau d \mathbf{y} d \mathbf{x} .
$$

\section{Conclusion}

In the paper, we obtain a mathematical model for the description of a non-local ferromagnetic material through the use of fractional derivatives. We have generalized the constitutive equation of a ferromagnetic material with memory to include dependence on the magnetic field in a wide neighborhood of each point $\mathbf{x}$ of the body. Using this approach, we are able to model materials with fading memory and also non-local properties.

\section{References}

[1] M. Fabrizio and A. Morro, Electromagnetism of continuous media: mathematical modelling and applications, Oxford University Press, Oxford UK, (2003)

[2] M. Caputo, Linear models of dissipation whose Q is almost frequency independent, part II, Geophys. J. R. Astron. Soc. 13, 529-539 (1967).

[3] M. Fabrizio, B. Lazzari and R. Nibbi, Existence and stability for a visco-plastic material with a fractional constitutive equation, Math. Meth. Appl. Sci. 40, 6306-6315 (2017).

[4] M. Caputo and M. Fabrizio, A new definition of fractional derivative without singular kernel, Progr. Fract. Differ. Appl. 1, 73-85 (2015).

[5] S. M. Mikki and A. Kishk, Electromagnetic wave propagation in nonlocal media: Negative group velocity and beyond, Progr. Electr. Res. B 14, 149-174 (2009).

[6] M. Fremond, Non-smooth thermomechanics, Springer, Berlin, 2002.

[7] J. M. Golden and G. A. C. Graham, Boundary value problems in linear viscoelasticity, Springer, Berlin, 1988. 